\title{
Large Berry phases in layered graphene
}

\author{
R. Englman* \\ Soreq NRC, Yavne 81800, Israel \\ T. Vértes:i \\ Institute of Nuclear Research of the Hungarian Academy of Sciences \\ H-4001 Debrecen, P.O. Box 51, Hungary
}

(Dated: November 4, 2018)

\begin{abstract}
Brillouin zones of graphene systems possess Dirac points, where band degeneracies occur. We study the variety of (and large magnitude) phases that the electronic states can acquire when a uniform time-dependent electric field carries the electrons around one or more Dirac points in a nonconcentric fashion. An experimentally accessible determination of excess Berry phases is proposed involving the Zitterbewegung of electronic current near an orthogonality point in adiabatic motion.
\end{abstract}

PACS numbers: 73.22.-f, 73.63.-b, 81.05.Uw, 03.65.Vf

\section{INTRODUCTION}

The recent research interest in graphene owes much to the remarkable electronic properties near the chargeneutrality, Dirac points (DP's, conical intersections or linear electronic degeneracies) at the $\mathbf{K}$ and $\mathbf{K}^{\prime}$-points in the Brillouin zone $\frac{1,2,3,4,5,6}{6}$. Also several studies devoted to graphene layers of various thicknesses have appeared recently $7,8,9,10,11,12,13$. Based on the earlier elucidation of the Brillouin-zone structure in Refs. 14, 15 and the more recent works in Refs. 16. 19, in bilayered graphene one centrally and three trigonally arranged DP's around the $\mathbf{K}$-type points can be located. The electronic motion is described by a Hamiltonian that includes interactions between different sites in either plane and between the two planes and expressed the coupling between four electronic bands. An approximate and simplified model using two $2 \times 2$ Hamiltonians (for the $\mathbf{K}$ and $\mathbf{K}^{\prime}$-points, respectively) was given in Ref. 8 . The repercussions of different Hamiltonians on the zero energy minimal conductivity were discussed in Ref. 12, where the effect of the trigonal distortion was found to be significant, whereas the simplified Hamiltonian of Ref. 10 led to results similar to those of the four band model.

Another development regarded the Berry phase ${ }^{20,21}$ acquired upon circling around the DP's in the kplane $3,7,8,13$. It was assumed in these works that a circling around each point leads to an added value of $\pm \pi$ to the geometric phase, with the sign depending on derivatives in the neighborhood of the intersection points. (The sign of the Berry phase encountered in the molecular physics, electron-vibration context was determined in Refs. 22, 23.) It is the purpose of the present work to show that when a Berry phase is actually created in a physical, adiabatically cyclic process, then the magnitude of the phase will differ from the assumed value of $\pm \pi$ and is more correctly $(2 n+1) \pi$, where $n$ is a signed integer or zero. The above result holds for a single DP, when the circling is performed in a non-concentric manner (and, likewise, when the circling is around an odd number of intersections). Analogously, when the circle is around two or an even number of intersection points the assumed result of $\pm 2 \pi$ or zero is superseded by $2 n \pi$ (with $n$ taking values, as before). These predictions are based on a proper treatment of the time-dependent adiabatic process, which requires near an orthogonality point a formalism that involves a correction term beyond the extreme adiabatic limit $24,25,26$. (At an "orthogonality point" the momentary wave function has zero overlap with the starting wave function. The integer $n$ is related to the varying speed of the electron along its cyclic path. This is quantified by the parameter $n^{\prime}$, named "the inverse relative slowness" and defined below in equation (19). In the present context and formalism the extreme adiabatic limit is defined by the vanishing of the ratio $\epsilon$ in equation (14) for all times, while n' in equation (18) is finite and possibly numerically large. A conventional treatment would first neglect the second and third terms in equation (16) and then obtain a $\pm \pi$ Berry phase from the sign change of the first term. We retain the remaining terms and calculate the acquired phases at the several zeros near the orthogonality point, neglecting the small terms only after exiting from the orthogonality neighborhood.)

Now it is true that Berry phases are usually written modulo $2 \pi$, and are gauge invariant only under conditions of complete cycling, but the effect of the extra $|n| \neq 0$ shows up even before completion of the cycling, as an open path phase $\underset{27}{ }$, and can in principle be measurable. (The extra acquired phase is gauge invariant $\underline{24,25,26}$. Experimental verification of the extra open path phase has been proposed before ${ }^{28}$.) An essential point (not present in previous electron-nuclear settings) is that in graphene it is technically feasible to control the phase acquisition by varying the electron concentration and by an electric field. The former is achieved by the manipulation of the (time-constant) gate voltage (through the substrate) and the latter by a well designed application of a timevarying uniform in-plane electric field that guides the electronic motion through the "acceleration theorem" (of which more in the sequel). Application of a time independent electric field was treated in Ref. 29. In the present setting of the adiabatic change in the electronic wave vec- 
tor, the magnitude of the applied electric field would be of the order of $10^{4} \mathrm{~V} / \mathrm{m}$ or less; this is rather less than the value $10^{7} \mathrm{~V} / \mathrm{m}$ proposed in the preceding reference, appropriate to conditions that are not adiabatic.

Although in this work we do not detail the experimental aspects of the phase acquisition, we do propose and investigate theoretically an entirely new method for the experimental observation of the Berry phase, feasible for high Berry phase values. (In our future mentioning of the phase, the dynamic phase of Ref. 20 is understood to be subtracted from the total wave-function phase. We return to the dynamic phase in section VII]) This is made possible during the acquisition of the phase in the vicinity of the orthogonality point and is measurable through the Zitterbewegung (ZB) of the electronic motion (current). $\mathrm{ZB}$ in graphene related materials was the subject of several works $33,34,35,36$. (Also related is reference 37.) It is shown in section VI that in a adiabatically time developing eigenstate, $\mathrm{ZB}$ is observable near the orthogonality points. The time duration of the $\mathrm{ZB}$ is associated with the inverse relative slowness parameter. In the present context where adiabatic changes are considered, the duration for which $\mathrm{ZB}$ has to be observed is expected to be rather large, of the order of $10^{3}$ fs or more. (Restrictions on the duration of the $\mathrm{ZB}$ by the finite width of the electronic wave-packet were previously considered in Refs. $34,36,38$.)

\section{GRAPHENE-BASED HAMILTONIANS}

\section{A. Monolayer graphene}

This is a zero-gap semiconductor, whose zerotemperature electronic properties in the undoped or slightly doped form arise from the neighborhood of hexagonally arranged $\mathbf{K}$-points (the Dirac points, DP) $\frac{5}{5}$. Here the valence and conduction bands meet in a conical intersection. The Hamiltonian, expressed by means of the planar quasi-particle momenta $\left(k_{x}, k_{y}\right)$ measured from a $\mathbf{K}$-point, have the form of a two-dimensional Dirac-Hamiltonian

$$
H=v_{F}\left(\begin{array}{cc}
k_{x} & k_{y} \\
k_{y} & -k_{x}
\end{array}\right),
$$

where a real representation was adopted for the electronic band states and $v_{F}$ is the Fermi velocity (about $10^{6} \mathrm{~m} / \mathrm{s}$ ). The off-diagonal, complex Hamiltonian commonly used in the graphene literature is $M^{\dagger} H M$, having applied the unitary transformation matrix

$$
M=\frac{1}{\sqrt{2}}\left(\begin{array}{cc}
1 & 1 \\
-i & i
\end{array}\right)
$$

to the above Hamiltonian $\mathrm{H}$.

For future use we note that for momenta $|k|$ whose cyclic time rate of change is represented by $\omega$, the requirement of adiabaticity is given in a monolayer graphene by

$$
v_{F}|\mathbf{k}| \gg \omega \text {. }
$$

\section{B. Graphene bilayer}

Four electronic bands describe the salient properties. A concise form of the 4-band Hamiltonian for an electron in a $\mathbf{k}$ (wave vector) state near the $\mathbf{K}$ and $\mathbf{K}^{\prime}$-points was written out in Ref. 12. (Eq. (1) there.) An approximate 2-band Hamiltonian proposed by Ref. 8 can be written in a real electronic state representation and following the real-matrix notation used in Refs. 24-26 as

$$
H_{\mathbf{K}}(\tilde{\mathbf{k}})=\left(\begin{array}{cc}
-U(\tilde{\mathbf{k}}) & V(\tilde{\mathbf{k}}) \\
V(\tilde{\mathbf{k}}) & U(\tilde{\mathbf{k}})
\end{array}\right)
$$

for (e.g.) the $\mathbf{K}$-point. Here the designation $\tilde{\mathbf{k}}$ is used for the reduced in-plane wave-vector measured from the K-point.

$$
\tilde{\mathbf{k}} \equiv \frac{\mathbf{k}}{k_{0}}=\frac{\left(k_{x}, k_{y}\right)}{k_{0}} \equiv\left(\tilde{k}_{x}, \tilde{k}_{y}\right),
$$

where $k_{0}=\frac{2 \gamma_{1} \gamma_{3}}{\sqrt{3} a \gamma_{0}^{2}}$ having introduced the intra-layer coupling strength $\gamma_{0}$ between the basis atoms A and B within the first layer and between A' and B' within the second layer, the vertical interlayer A-B' coupling strength $\gamma_{1}$, the (weaker) diagonal interlayer A'-B coupling strength $\gamma_{3}$ and the honeycomb lattice constant $a$. Numerical values that have been accepted for these parameters are $\gamma_{0}=3.16 \mathrm{eV}, \gamma_{1}=0.39 \mathrm{eV}, \gamma_{3}=0.315 \mathrm{eV}, a=0.246 \mathrm{~nm}, k_{0}=$ $0.05775 \mathrm{~nm}^{-1}$. The matrix elements are

$$
\begin{aligned}
U(\tilde{\mathbf{k}}) & =e_{0}\left[\tilde{k}_{x}-\left(\tilde{k}_{x}^{2}-\tilde{k}_{y}^{2}\right)\right], \\
V(\tilde{\mathbf{k}}) & =e_{0}\left[\tilde{k}_{y}+2 \tilde{k}_{x} \tilde{k}_{y}\right],
\end{aligned}
$$

with an overall coupling energy $e_{0} \equiv \gamma_{1}\left(\frac{\gamma_{3}}{\gamma_{0}}\right)^{2}=3.87$ meV. For a $\mathbf{K}^{\prime}$-point the signs of the first term in $U(\tilde{\mathbf{k}})$ and of the second term in $V(\tilde{\mathbf{k}})$ need to be changed.

\section{MOTION OF ELECTRONS}

When subject to an electric field $\mathbf{E}$, the wave vector of an electron (with charge $-e$ ) changes in time $(t)$ according to the "acceleration theorem"30,31

$$
\frac{d \mathbf{k}}{d t}=-\frac{e}{\hbar} \mathbf{E}
$$

A spatially uniform and time varying electric field is expressed in terms of a vector potential $\mathbf{A}(t)$ through

$$
\mathbf{E}=-\frac{\partial \mathbf{A}(t)}{\partial t}
$$

giving

$$
\mathbf{k}(t)=\frac{e}{\hbar} \mathbf{A}(t)
$$

apart from an initial value of the wave vector. Thus, the above Hamiltonians are to be understood as functions of the vector potential or of the time integral of the 
externally applied electric field. This will be the meaning attached to the independent variables ( $\mathbf{k}$ or $\tilde{\mathbf{k}}$ ) in the Hamiltonians, though (for simplicity of notation) we shall continue to write them as functions of the timedependent planar wave-vectors, rather than that of $\mathbf{A}$. In the sequel we shall assume the following time dependence for the moving reduced wave vector:

$$
\begin{aligned}
& \tilde{k}_{x}(t)=a \cos (\omega t)+c, \\
& \tilde{k}_{y}(t)=b \sin (\omega t),
\end{aligned}
$$

with constants $a, b$ and $c$ and a period of $T=2 \pi / \omega$.

\section{GEOMETRIC PHASE OF THE WAVE FUNCTION COMPONENT}

Solutions of the time dependent Schrödinger equation, written for a general form of a periodic $2 \times 2$ Hamiltonian as in equation (44) and valid in the adiabatic limit, were given in Refs. 24.26, with special regard to the geometric phase acquired near an "orthogonality time" $t_{v}$. This is the instant at which the initially engaged component of the quasi-spinor vanishes, or the state becomes orthogonal to the initial state. There may be several such instants, depending on the path taken by the time dependent parameters. As already remarked in the Introduction, the solutions of the above cited works went one approximation (in the adiabatic parameter, to be defined shortly) beyond the formal adiabatic wave function. The instantaneous energy and twice the mixing angle appropriate to the Hamiltonian in equation (4) (and analogously to that in equation (10) ) are

$$
\begin{aligned}
W(t) & \equiv \sqrt{U(\tilde{\mathbf{k}}(t))^{2}+V(\tilde{\mathbf{k}}(t))^{2}}, \\
\chi(t) & \equiv \arctan \frac{V(\tilde{\mathbf{k}}(t))}{U(\tilde{\mathbf{k}}(t))}
\end{aligned}
$$

The (small) adiabaticity parameter is in terms of these

$$
\epsilon(t)=\frac{\dot{\chi}(t)}{W(t)}
$$

We now turn to the solution of the time dependent Schrödinger equation in the form

$$
i \frac{\partial}{\partial t}\left(\begin{array}{c}
f(t) \\
g(t)
\end{array}\right)=\left(\begin{array}{cc}
-U(t) & V(t) \\
V(t) & U(t)
\end{array}\right)\left(\begin{array}{l}
f(t) \\
g(t)
\end{array}\right)
$$

with the initial condition for an energy eigenstate $f(t=$ $0)=1, g(t=0)=0$. In Refs. 24, 26 we have obtained (after the removal of the dynamic phase factor) the following component amplitudes of the wave function in the neighborhood of an orthogonality point $t_{v}$ as

$$
\begin{aligned}
f(t)= & -\frac{1}{2} \dot{\chi}\left(t_{v}\right)\left[\left(t-t_{v}\right)\right. \\
& \left.-\frac{i}{2\left|W\left(t_{v}\right)\right|}\left(1-\pi n^{\prime}\left(t_{v}\right) e^{-2 i\left|W\left(t_{v}\right)\right|\left(t-t_{v}\right)}\right)\right] \\
& +\frac{1}{2} \pi \phi\left(t_{v}\right) \epsilon\left(t_{v}\right), \\
g(t)= & -\sin \frac{1}{2} \chi\left(t_{v}\right)=-1 .
\end{aligned}
$$

The expressions are correct to the order of $\left|\dot{\chi}\left(t_{v}\right)\left(t-t_{v}\right)\right|^{2}$.

$$
n^{\prime}\left(t_{v}\right) \equiv \pi^{-1} \frac{\epsilon(0)}{\epsilon\left(t_{v}\right)}
$$

which ratio compares the slownesses (or adiabaticities, defined in equation (15)) at the starting points and at the orthogonality point. It is a key quantity in the phase acquisition phenomenon, as we shall describe below. In contrast, the last term on the right of equation (17) is of little importance. (This has been confirmed both theoretically and by numerical computation). In it $\phi\left(t_{v}\right)$ is the fractional part of the energy integral $2 \int_{0}^{t_{v}} W(t) d t$. (See Eq. (31) in reference 25.) The energy (or frequency) $2\left|W\left(t_{v}\right)\right|$ in the complex exponential is the energy separation between the two eigenstates near the orthogonality point and will show up in our treatment of the electronic motion (or current) in section VI as a Zitterbewegung.

We shall now describe the geometric phase acquisition process in terms of the minority component $f(t)$ in equation (17). Were it only the first term in $f(t)$, a phase of $-\pi$ would accrue upon traversing the zero at $t=t_{v}$ from $t<t_{v}$ to $t>t_{v}$. This phase is continuously carried in $f(t)$ and would remain with the wave function also when a full revolution is made at which instant the $g(t)$ component becomes again zero. Thus, $-\pi$ is indeed the final result (the Berry phase) as long as $n^{\prime}\left(t_{v}\right)$ is smaller than or of the order of 1 . However, when $n^{\prime}\left(t_{v}\right)$ is numerically large, there are further zeros in the complex t-plane, which are all located in the lower half of the complex t-plane (at shallow depths of the order $\left.1 /\left|W\left(t_{v}\right)\right|\right)^{24,25,26}$. Looking now for the moment at the behavior of the real part of $f(t)[\operatorname{Re} f(t)]$, with algebraically increasing real t, as this passes each adjacent (complex) zero, $\operatorname{Re} f(t)$ acquires a further $-\pi$ phase. This is also how the full function $f(t)$ acquires its phase, with the role of the imaginary part being to ensure that the phase of $f(t)$ changes in a smooth, continuous way. The rate of $\pi$ - acquisitions is clearly $2\left|W\left(t_{v}\right)\right| / \pi$ (a constant for a given orthogonality point $\left.t_{v}\right)$.

How many (complex) zeros are there in the asymptotic limit of large $\left|n^{\prime}\left(t_{v}\right)\right|$ ? Taking into account that the modulus of the complex exponential in equation (17) is unity, we clearly see that the complex zeros arise only as long as $\left|t-t_{v}\right| \leq \frac{\pi n^{\prime}\left(t_{v}\right)}{2\left|W\left(t_{v}\right)\right|}$, since otherwise the first term will dominate the function and zeros are not possible. Taking into account the previously obtained value $\left|2 W\left(t_{v}\right)\right| / \pi$ for the 
rate of acquisition of $\pi$ 's, we see that the number $n\left(t_{v}\right)$ (an integer!) of acquired $\pi$ phases is for either positive or negative $t-t_{v}$ 's about $2 n^{\prime}\left(t_{v}\right)$. [The same result would be obtained by counting the number of loops around the origin of $f(t)$, since each (anti-clockwise) loop contributes a $2 \pi$ phase and each loop around the origin implicates two zeros of $\operatorname{Re} f(t)$ (as well as of $\operatorname{Im} f(t)$ ).]

The net result is then that the acquired Berry phase around $t=t_{v}$ is

$$
\left[1+2 n\left(t_{v}\right)\right] \pi
$$

where $n\left(t_{v}\right)$ is a signed integer (or zero) close to the ratio $n^{\prime}\left(t_{v}\right)$ defined in equation (15). $n^{\prime}$ is named the inverse slowness ratio and examples taken from molecular degeneracies have shown that it is unity for concentric circular motion around a single intersection point (and, in the case of several intersection points, for concentric circular motion around one intersection point provided the motion is sufficiently far away from other intersections). However it can be large for circling that starts close to an intersection point and is not concentric with it $24,25,26,32$. For graphene layers similar results for the phase have now been found and are shown in a set of figures. These are obtained by solving the time dependent Schrödinger equation and in them the acquired phases agree very closely with $\left(2 n^{\prime}+1\right) \pi$, in which $n^{\prime}$ is given by the formula in equation (19). This depends only on quantities contained in the Hamiltonian. The case of (monolayer) graphene, with essentially isolated DP's (the $\mathbf{K}$ and $\mathbf{K}^{\prime}$ points), is formally identical to the single degeneracy results shown in section 3 of reference 32 and will not be reproduced here although monolayer graphene may be the more convenient candidate for the experimental verification of the theory. (However, the one order of magnitude lower value of $W\left(t_{v}\right)$ in bilayer graphene than in monolayer may make the oscillations easier to monitor.)

In a graphene bilayer we have a DP at each $\mathbf{K}$ (and $\mathbf{K}^{\prime}$ ) with three satellite DP's, trigonally situated around each $K$-type point. Thus, this is a new situation; also for the reason that there exist for it both two and four dimensional Hilbert space descriptions ${ }^{10,12}$. The Hamiltonian in the former description is shown in equation (6) and equation (7); some essential features of the energy contours are shown in Fig. 1.

We begin by showing results for a contour that starts close to and just outside a trigonal DP and makes a beeline with a path ultimately skirting round the central DP. [In Koshino and Ando's reference 10 figure 2, the path might start at an energy of about (or smaller than) $0.5 e_{0}$ (roughly equivalent to an electron density below $4 \times 10^{10} \mathrm{~cm}^{-2}$ ) or, otherwise, at $k_{x} \leq 1.2 k_{0}, k_{y}=0$ and then return so as to come down beyond the origin of $\mathbf{k}$, the K-point.] A full contour of this type is not expected to change the sign of the wave-function (with the dynamic phase disregarded), since two DP's are encompassed by it. In this case one obtains the variation of the $2 n$ part of the phase near an orthogonality point,

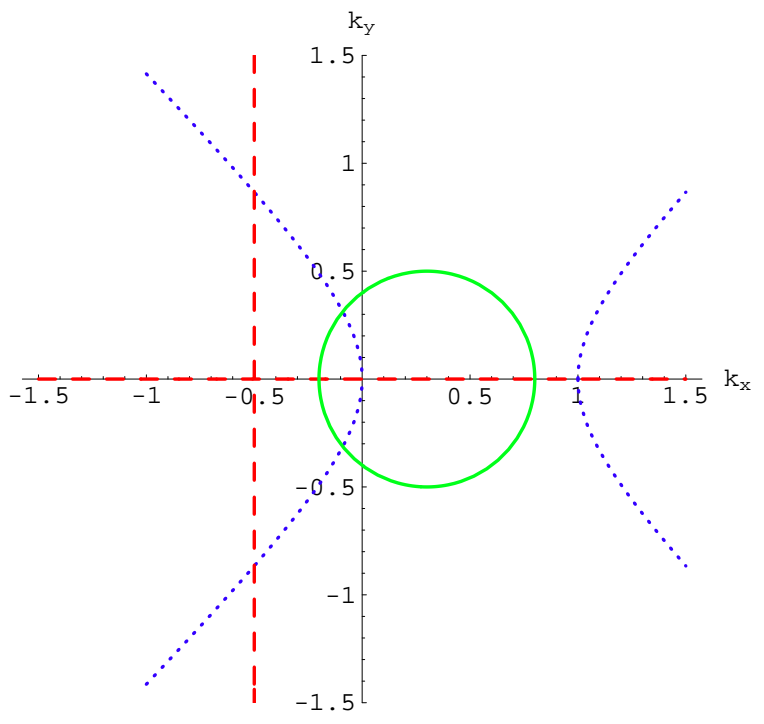

FIG. 1: (color online). Contours for bilayer graphene (in the two state-representation). Broken curves (in red): The potential $V=0$ (in the Hamiltonian of equation (4)). Dotted curve (in blue) : The potential $U=0$. The circle (in green) denotes an evolution path. The positions of the Dirac points are at the crossings of the broken and dotted curves. In the text, the evolution starts at a positive value of the abscissa. Orthogonality points $t_{v}$, where $\chi=\pi$, are where the path intersects the broken (red) curve. For the evolution path shown (circling radius $=0.5$, shift $=0.3$ ), there is only a single orthogonal position $t_{v}$ on the circular path, which is located on the negative abscissa. For circles shifted to the left it is possible to have two more $t_{v}$ 's.

which is of interest. In figure 2 we show (with open circle symbols) the resulting $n^{\prime}$ in a rather simple circling situation, when the contour starts to the right of the central DP and cycles anti-clockwise round it and inside the trigonal DP's. An orthogonality point $t_{v}$ is met at about (but not at exactly) half a full circle. Here the (open path) geometric phase makes a jump, whose magnitude is close to the ratio $n^{\prime}\left(t_{v}\right)$ defined above. For a circular contour around the central DP (which is the path here taken) the magnitude of $n^{\prime}$ depends on the distance of the starting point (located between the rightmost and the central DP's). As figure 2 shows, $\left|n^{\prime}\right|$ is large for contours starting close to the central DP and decreases as the starting point recedes only to become again large when the starting point is precisely at half way to a trigonal DP. This finding arises from the presence of several DP and is absent for a Brillouin zone with a single DP. The triangles in the same figure show, for comparison, the phase jumps as obtained by numerically solving the time dependent Schrödinger equation (the subject of the next subsection). 


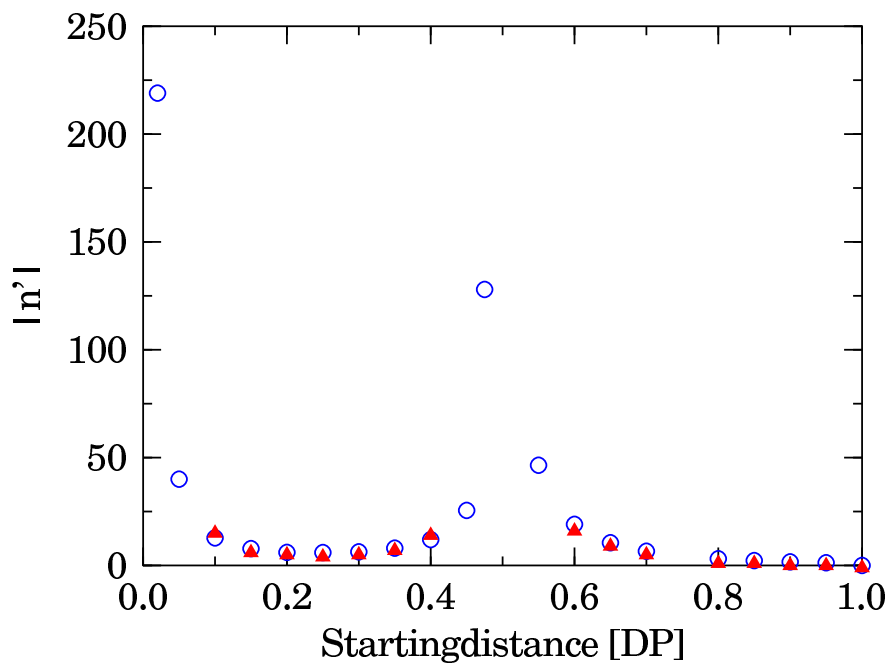

FIG. 2: (color online). Inverse relative slowness parameter $n^{\prime}$ for adiabatic circling plotted by open circles (in blue) against the starting distance from the central Dirac point (DP) in the direction of a trigonal DP (given in units [DP], measuring the distance of the trigonal DP from the central one). $n^{\prime}$ is approximated by the integer $n$ in the Berry phase $(2 n+1) \pi$ acquired at an orthogonality point $t_{v}$ during circling. A circular path with radius $0.5[\mathrm{DP}]$ was assumed. The inverse slowness parameter $n^{\prime}$ is large when the path starts near the central DP or (numerically) at just half way to the trigonal DP. (Here $n^{\prime}$ turns negative.) The triangles (in red) show the results of numerically computing the phase jumps. Some triangles are missing because of numerical difficulties in achieving convergence.

\section{NUMERICAL VERIFICATION OF THE "INVERSE RELATIVE SLOWNESS" PREDICTION}

In Refs. $24-26$ the Berry-phase was associated (through what we regard as the proper treatment of the time dependent Schrödinger equation (TDSE) in the near adiabatic limit) with the quantity $\left(2 n^{\prime}+1\right) \pi\left(\right.$ or $\left.2 n^{\prime} \pi\right), n^{\prime}$ being (as described above) the inverse relative slowness. In the quoted articles the theory was verified by numerical solution of the TDSE in the adiabatic limit, in the context of nuclear motion in electronically degenerate molecules. In the present graphene context (which differs from the molecular setting in essential physical, and some formal, details) the numerically obtained phase jump values $n$ have been plotted in Fig. 2 with triangles. These show good agreement with the values (open circles) obtained algebraically. Where the triangles are missing, this is due to numerical difficulties in achieving convergence.

An analogous verification has been performed by solving the TDSE for a graphene bilayer Hamiltonian, including a periodic, time dependent electric field $E(t)$ and calculating at each instant $t$ the phase of the wave function. In figure 3, the phase development is shown for one full cycle of the electric field and the detail for the
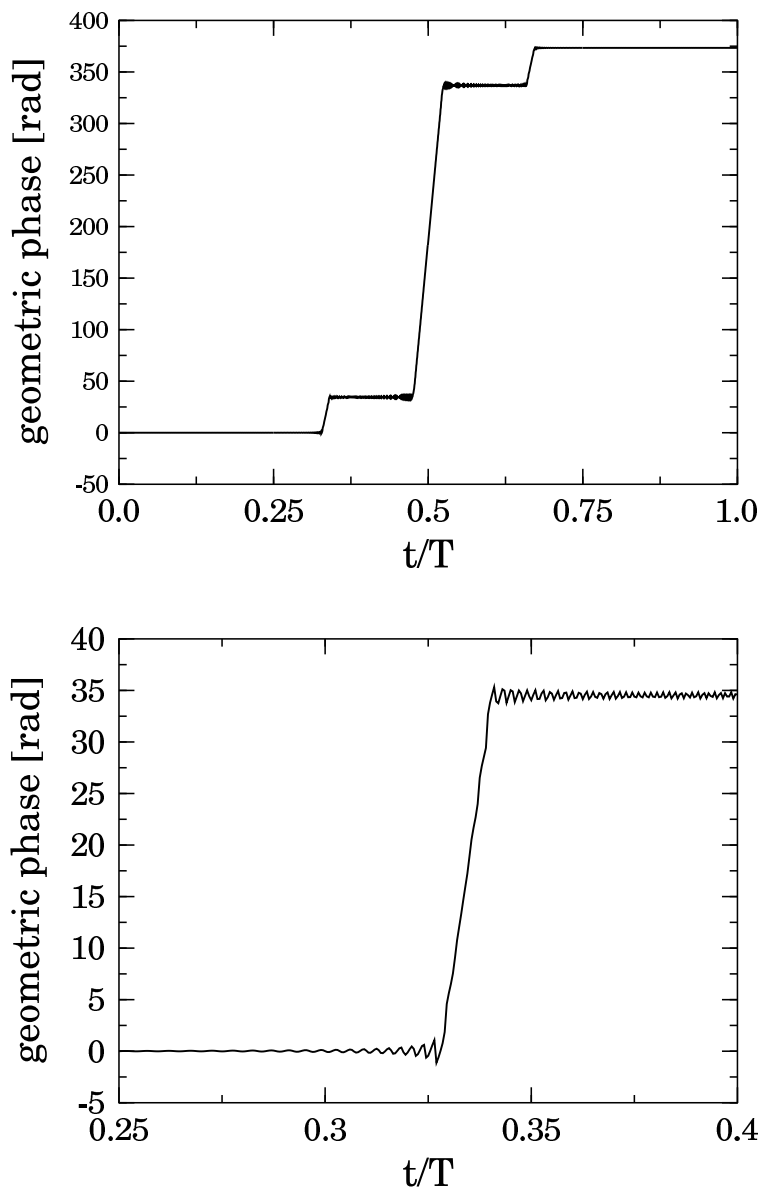

FIG. 3: Adiabatic phase evolution in bilayered graphene (obtained from numerical solution of the time dependent Schrödinger equation within the two-state formalism). The adiabatic revolution period $T$ is $2 \pi \times 10^{5}$ in inverse units of the Fermi velocity times the DP distance. Circular cycling meets three orthogonality points along its path (as explained in the caption of Fig. (1). In the upper drawing, at these points steep rises take place in the phase, with the middle one (on the left-part of the abscissa in figure 1) being the longest rise. The near-horizontal wavy part becomes straightened out, as the motion becomes more adiabatic. In the lower drawing the first $t_{v}$ region (above the $k_{x}$ axis) is enlarged.

first step. The non-constant, rising parts of the phase near the orthogonality points will be the subject of the section on "Currents".

The drawings in figures 1 1 3 have been based on the two-state (approximate) description of bilayer graphene. In figure 4 we compare the component phases obtained from forward integration of the time dependent Schrödinger equation for the four-state with that for the approximated two-state description $\underline{10}$. There are no differences visible to the naked eye, which we interpret as a satisfactory test for the robustness of the adiabatic theory formulated in our previous papers. 

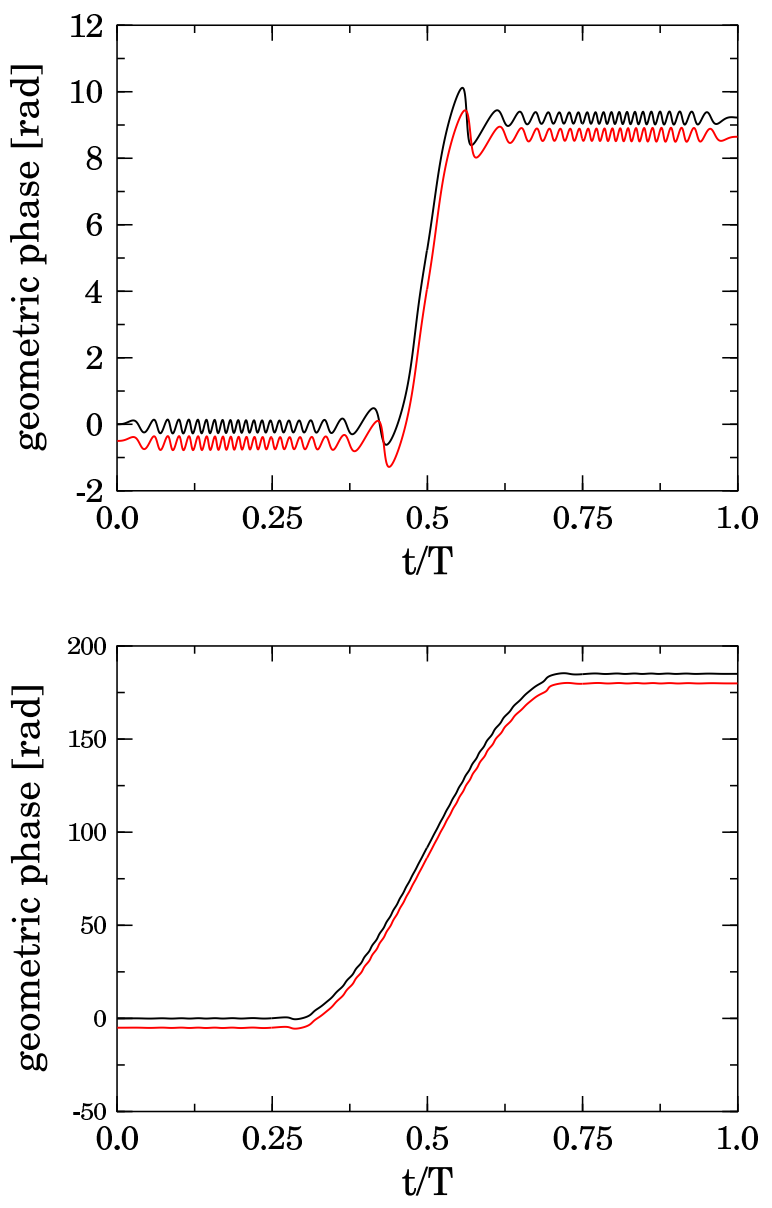

FIG. 4: (color online). Comparison of the Berry phase behaviors of the four- and two-state models in graphene bilayer. Curves for the four state model are slightly displaced downward, for clarity. Two different starting positions to the right of the central Dirac point (DP) were chosen, expressed in units of the distance to the right-most DP . Upper frame starting position: 0.8. Lower frame starting position: 0.25 . The adiabatic revolution period $T$ is $2 \pi \times 10^{4}$ in inverse units of the Fermi velocity times the DP distance. Note the different ordinate scales.

\section{ZITTERBEWEGUNG (ZB) OF THE CURRENT}

The equation of motion for the Heisenberg position operator $\mathbf{r}(t)$ of an electron is $i \dot{\mathbf{r}}(t)=-[H, \mathbf{r}(t)]$. The current is $\mathbf{j}(t)=-e \dot{\mathbf{r}}(t)$. We shall evaluate this for a monolayer graphene near its $\mathbf{K}$ point where the expressions are quite simple, rather than for a graphene bilayer, for which the results are analogous, but more complicated. Inserting the Hamiltonian from equation (11) one obtains immediately the velocity components as

$$
\dot{\mathbf{r}}(t) \equiv \dot{x}(t) \hat{\mathbf{i}}+\dot{y}(t) \hat{\mathbf{j}}=v_{F}\left(\begin{array}{cc}
\hat{\mathbf{i}} & \hat{\mathbf{j}} \\
\hat{\mathbf{j}} & -\hat{\mathbf{i}}
\end{array}\right)
$$

The observable, expectation value of the velocity is the expectation value over the wave function, namely,

$$
<\dot{\mathbf{r}}(t)>\equiv v_{F}\left(f^{*}, g^{*}\right)\left(\begin{array}{cc}
\hat{\mathbf{i}} & \hat{\mathbf{j}} \\
\hat{\mathbf{j}} & -\hat{\mathbf{i}}
\end{array}\right)\left(\begin{array}{c}
f \\
g
\end{array}\right),
$$

where the stars denote complex conjugates. Two situations need now to be considered.

\section{A. Electronic motion during most of the contour path}

\section{Extreme adiabatic limit, $\epsilon \rightarrow 0$}

Here

$$
\left(f^{*}, g^{*}\right)=(f, g)=\left(\cos \frac{1}{2} \chi(t),-\sin \frac{1}{2} \chi(t)\right)
$$

in terms of the mixing angle $\frac{1}{2} \chi(t)$, which effects the diagonalization of the $2 \times 2$ Hamiltonian. For the monolayer Hamiltonian in equation (11) this is defined through $\chi(t) \equiv \arctan \frac{V}{U}=-\arctan \frac{k_{x}(t)}{k_{y}(t)}$, so that it is possible to express the momentum components as $k_{x}(t)=$ $|\mathbf{k}| \cos \chi(t), k_{y}(t)=-|\mathbf{k}| \sin \chi(t)$, where the time dependence arises from the applied vector potential as in equation (10). Then equation (22) evaluates to

$$
<\dot{\mathbf{r}}(t)>=v_{F}[\cos \chi(t) \hat{\mathbf{i}}-\sin \chi(t) \hat{\mathbf{j}}],
$$

which merely reaffirms the equivalence of the time behaviors of the momentum and velocity vectors for a completely adiabatic motion along most of the pathway.

\section{First order adiabatic correction}

When the wave function components are expanded correct to the order of the small adiabatic parameters $\epsilon(t)$, one obtains the terms describing the $\mathrm{ZB}$ of the electronic velocities.

$$
\begin{aligned}
& <\dot{x}(t)>_{Z B}=-\frac{v_{F} \epsilon(0)}{2} \sin \chi(t) \sin 2 I(t), \\
& <\dot{y}(t)>_{Z B}=-\frac{v_{F} \epsilon(0)}{2} \cos \chi(t) \sin 2 I(t),
\end{aligned}
$$

where we have denoted the integral over energy as

$$
I(t) \equiv \int_{0}^{t} d t^{\prime} W\left(t^{\prime}\right)
$$

There may be some practical difficulties in the observation of this motion as it is superimposed on a larger motion shown in equation (24).

\section{B. Near the orthogonality point $t_{v}$}

Here the eigenstate component amplitudes $(f, g)$ take the time-dependent forms shown in equation (17) and 
equation (18). Substitution into equation (22) shows that in the adiabatic limit the $x$-component moves with a uniform speed of $v_{F}$. However, the $y$-component of the electron has the value

$$
\dot{y}(t)= \pm 2 v_{f} \operatorname{Ref}(t) .
$$

As discussed in section IV this is a small quantity, of the order of the small adiabaticity parameter $\epsilon\left(t_{v}\right)$ which oscillates with the period characteristic of ZB, given in the present case by $\pi / W\left(t_{v}\right)$. The eigenstate oscillations take place for a time interval of about $\frac{n^{\prime}}{W\left(t_{v}\right)}$, placed symmetrically about $t_{v}$. This interval can be quite large in case of strongly non-concentric circling, for which the relative inverse slowness $n^{\prime}$ (defined in equation (19)) has large values (e.g., of the order of $\left.10^{2}\right) \underline{39}$.

\section{SUBTRACTION OF THE DYNAMIC PHASE}

Results in the previous sections have the following implication (again phrased for simplicity's sake for the monolayer graphene case, section ЏA): For a given radius of circling $k_{c}$ around a $\mathbf{K}$-point the acquired Berry phase can be increased by diminishing the distance $\Delta k$ between the starting point and the center of the circular path. The increase in the Berry phase is about $2 \pi$ times $n^{\prime}$ (the relative inverse slowness) which is unity when the distance $\Delta k=k_{c}$ and increases beyond bounds as $\Delta k \rightarrow 0$. A hypothetical direct experimental determination of the augmented phase would then likely perform two measurements at or close to these two limits.

Since the above theory relates to the Berry phase, while the total acquired phase includes also the dynamical phase (designated $\phi_{D}$ ), it is purposeful to estimate the latter. It was shown in Refs. 24-26 that in the adiabatic limit [equation (3) above] this is simply given by the time integral of the instantaneous energy. Then for a uniform circular motion in the $\mathbf{k}$-plane

$$
\begin{aligned}
& \phi_{D}\left(k_{c}, \Delta k\right) \\
& =v_{F} \int_{0}^{2 \pi / \omega} \sqrt{\left.\left(k_{c} \cos \omega t\right)-\Delta k\right)^{2}+\left(k_{c} \sin \omega t\right)^{2}} d t .
\end{aligned}
$$

This elementary integral has the following values in the two limiting cases discussed above:

$$
\begin{aligned}
\text { concentric cycling: } \phi_{D}\left(k_{c}, k_{c}\right) & =2 \pi \frac{v_{F}\left|k_{c}\right|}{\omega}, \\
\text { touching: } \phi_{D}\left(k_{c}, 0\right) & =2 \sqrt{2} \frac{v_{F}\left|k_{c}\right|}{\omega} .
\end{aligned}
$$

These dynamic phase values can be subtracted from the observed total phase to obtain the Berry-phase. From our direct computations of the acquired total phase by the wave function, for situations such as shown in Fig. 3 we find that for large $\left|n^{\prime}\right|$-values the phase is dominated by the Berry-phase. (The dynamic phases at the three phase jumps shown in that figure amount to $-20,-60,-20$ radians, respectively.)

\section{CONCLUSION}

This work has focused on two (interrelated) issues in the context of mono- and bilayered graphene.

First, (based on our past works on adiabatic cycling, which are only briefly recapitulated here) we have shown that large amplitude (and gauge-invariant) phases of the electronic wave-functions can be generated in twodimensional structures of the graphene-type. A conceptual experimental procedure involving a uniform time varying electric field has been outlined, though without our proposing detailed prescription. The key geometrical element is the non-concentric adiabatic path in $\mathbf{k}$-space around the $\mathbf{K}$ points.

The second issue is the association of the developmental stage of the large amplitude Berry phase (near the orthogonality points) with the Zitterbewegung of the electronic motion and its observational possibility. So far little attention has been given to the possibility of experimentally detecting the Berry phase in the neighborhood of an orthogonality point; though some theoretically oriented remarks regarding the abrupt nature of this developmental stage of the Berry phase have been made 40,41 . These remarks addressed the minimal $\pm \pi$ phase acquisitions near each orthogonality point, whereas our proposal for the observation of the phase by Zitterbewegung is for large Berry phases.

\section{Acknowledgments}

Thanks are due to Joshua Zak and Yizhak Yacoby for educating remarks. T.V. has been supported by a János Bolyai Grant of the Hungarian Academy of Sciences.

\footnotetext{
* Electronic address: englman@vms.huji.ac.il

† Electronic address: tvertesi@dtp.atomki.hu
}

${ }^{1}$ K.S. Novoselov, A.K. Geim, S.V. Morozov, D. Jiang, Y. Zhang, S.V. Dubonos, I.V. Griorieva and A.A. Firsov, Sci- 
ence 306, 666 (2004).

2 K.S. Novoselov, A.K. Geim, S.V. Morozov, D. Jiang, M.I. Katsnelson, S.V. Dubonos, I.V. Griorieva and A.A. Firsov, Nature 438197 (2005).

3 Y. Zhang, Y.-W. Tan, H.L. Stormer and P. Kim, Nature 438, 201 (2005).

4 M.I. Katsnelson, Materials Today, bf 10, 20 (2007).

5 A.K. Geim and K.S. Novoselov, Nature Materials 6, 183 (March 2007).

6 A.H. Castro Neto, F. Guinea, N.M.R. Peres, K.S. Novoselov and A.K. Geim, arXiv:0709.1163 (2007).

7 K.S. Novoselov, E. McCann, S.V. Morozov, V.I. Fal'ko, M.I. Katsenelson, U. Zeitler, D. Jiang, F. Schedin and A.K. Geim, Nature Physics 2, 177 (March 2006).

8 E. McCann and V.I. Fal'ko, Phys. Rev. Lett. 96, 086805 (2006).

9 B. Partoens and F.M. Peeters, Phys. Rev. B 74, 075404 (2006).

10 M. Koshino and T. Ando, Phys. Rev. B 73, 245403 (2006).

11 J.L. Manes, F. Guinea and M.A.H. Vozmediano, Phys. Rev. B 75, 155424 (2007).

12 J. Cserti, A. Csordás and G. Dávid, Phys. Rev. Lett. 99, 066802 (2007).

13 G.P. Mikitik and Yu. V. Sharlai, Phys. Rev. B 77, 113407 (2008).

14 J.W. McClure, Phys. Rev. 108, 612 (1957).

15 J.C. Slonczewski and P.R. Weiss, Phys. Rev. 109, 272 (1958).

16 N.M.R. Peres, F. Guinea and A.H. Castro Neto, Phys. Rev. B 73, 125411 (2006).

17 L. Brey and H.A. Fertig, Phys. Rev. B 73, 235411 (2006).

18 H. Min and A.H. MacDonald, Phys. Rev. B 77, 155416 (2008); arXiv:0806.2792 1 [cond-mat.mes-hall]

19 L. Malysheva and A. Onipko, Phys. Rev. Lett. 100, 186806 (2008).

20 M.V. Berry, Proc. Roy. Soc. (London) A 392, 45 (1984).

${ }^{21}$ K. Yu. Bliokh and Yu. B. Bliokh, Ann. Phys. (N.Y.) 319 13, (2005).

22 R. Englman and A. Yahalom, Acta Phys. Chim. Debrecina 34-35, 283 (2002).

23 T. Vértesi and E. Bene, Chem. Phys. Lett. 392, 17 (2004).

${ }^{24}$ R. Englman and T. Vértesi, J. Phys. B: At. Mol. Opt. Phys. 38, 2443 (2005).

25 T. Vértesi and R. Englman, Phys. Rev. A 73, 022103
(2006).

26 R. Englman and T. Vértesi, Phys. Lett. A 354, 196 (2006).

27 M. Mukunda and R. Simon, Ann. Phys. (N.Y.) 228, 205 (1993); A.K. Pati, Phys. Rev. A 52, 2576 (1995); S.R. Jain and A.K. Pati, Phys. Rev. Lett. 80, 650 (1998).

${ }^{28}$ R. Bhandari, Phys. Rev. Lett. 89, 268901 (2002).

29 J.C. Martinez, M.B.A. Jalil and S.G. Tan, "Particle-hole Hall effect in graphenelike structures", arXiv:0806.0222 v1 [cond-mat.mes-hall]

30 L.D. Landau and E. M. Lifshitz, Quantum Mechanics (Pergamon Press, London, 1958).

31 J. Zak, Solid State Physics 27, 1 (1972).

32 R. Englman, A. Yahalom and T. Vértesi, J. Mol. Struct. 838, 20 (2007).

33 M.I. Katsnelson, Eur. Phys. J. B 51, 157 (2006).

34 T.M. Rusin and W. Zawadzki, Phys. Rev. B 76, 195439 (2007).

35 J. Cserti and G. Dávid, Phys. Rev. B 74, 172305 (2006).

36 T.M. Rusin and W. Zawadzki, arXiv:0712.3590 [condmat.mes-hall] (21 Dec 2007).

37 J. Schliemann, D. Loss and R.M. Westervelt, Phys. Rev. Lett. 94, 206801 (2005).

38 J.A. Lock, Am. J. Phys. 47, 797 (1979).

39 Previously, Ref. 34 have given expressions for the current's ZB in graphene. However, as they note after their equations (3) and (9), their derivation is not for a developing adiabatic eigenstate, which is what we treat in this work, but for a non-stationary state. In another work ${ }^{35}$ operator expressions were given for the electronic coordinates in several systems, including monolayer and bilayer graphenes. Here again, when the expectation values of the ZB-terms are evaluated for an adiabatically evolving eigenstate, then all these vanish in the extreme adiabatic limit (cf. our treatment in section VIB). (This does not occur in the electron-positron framework, where the $z$-momentum is non-zero.) As shown in section VIB in graphene the leading contribution to the $\mathrm{ZB}$ term in the electron displacement is of order $\epsilon(0)$.

40 E. Sjöqvist and M. Hedström, Phys. Rev. A 56, 3417 (1997).

41 M. Baer, A. Yahalom and R. Englman, J. Chem Phys. 109, 6550 (1998). 